\title{
Evolution of vortex states under external magnetic field
}

\author{
Zung-Hang Wei ${ }^{\mathrm{a}, *}$, Ching-Ray Chang ${ }^{\mathrm{a}}$, Nickolai A. Usov ${ }^{\mathrm{b}}$, \\ Mei-Feng Lai ${ }^{\mathrm{a}}$, J.C. $\mathrm{Wu}^{\mathrm{c}}$ \\ ${ }^{a}$ Department of Physics, National Taiwan University, 1 Sec. 4 Roosevelt Road, Taipei, Taiwan 10617, Republic of China \\ ${ }^{\mathrm{b}}$ Troitsk Institute for Innovation and Fusion Research, 142092, Troitsk Moscow region, Russia \\ ${ }^{\mathrm{c}}$ Department of Physics, National Changhua University of Education, Changhua, Taiwan 500, Republic of China
}

\begin{abstract}
The behavior of one-, two-, and three-vortex states of submicron-sized elliptical elements under the influence of external magnetic field is investigated here by means of micromagnetic simulations. The total magnetization curves show abrupt changes at the nucleation or annihilation of vortices. The magnetization curves and critical field show significant dependence on the field direction with respect to the particle's long axis. (C) 2002 Elsevier Science B.V. All rights reserved.
\end{abstract}

Keywords: Vortex states; Micromagnetic simulations; Magnetization curves

Nano-sized ferromagnetic materials, which are energetically stable with a single domain structure, have recently been applied in the fabrication of spintronics devices, such as read heads of hard disk and magnetic memory, etc. On the other hand, in particles of larger sizes, domain structures usually appear to minimize the demagnetization energy. Because of the reduced magneto-static interaction between each bit in patterned media due to the virtue of flux-closure, the vortex states also have the potential in application [1]. Some theoretical and experimental results have been published [2-4] about the vortex state in elements of rectangular or other shapes with sharp corners. The existence of the sharp corners influences the magnetization structures of the particle. Hence it is helpful to investigate the properties of thin films of elliptical shape with no edge effect. In this article, we study the behavior of typical one-, two-, and three-vortex states of elliptical elements under an external field. The back-sweep process used for the transition between different vortex states is also found in our simulation. The vortex states show interesting behavior under external field due to the

\footnotetext{
*Corresponding author. Tel.: + 886-2-2363-9984.

E-mail address: crchang@phys.ntu.edu.tw (Z.-H. Wei).
}

shifting of the centers of the vortices in direction perpendicular to the magnetic field.

Micromagnetic simulations of submicron-sized elliptical elements are based on time integration of the Landau-Lifshitz-Gilbert (LLG) equation. The material parameters of the soft type Permalloy film are as follows: the exchange constant $C=2 A=2 \times 10^{-6} \mathrm{erg} /$ $\mathrm{cm}$, and the saturation magnetization $M_{\mathrm{s}}=800 \mathrm{emu} /$ $\mathrm{cm}^{3}$. The uniaxial anisotropy constant $K_{1}$ is assumed to be $10^{3} \mathrm{erg} / \mathrm{cm}^{3}$, which is actually too small to influence the total energy considerably. The size of the meshed cell is set to be $7.5 \mathrm{~nm}$, that is, smaller than half of the exchange length $17.7 \mathrm{~nm}$ of the Permalloy material in order, to insure the precision of our simulation. In our calculation it is assumed that the system has reached equilibrium state when the largest magnetization angular variation among all cubes is less than $10^{-6}$ radians. In our study, the thickness of the elliptical element is $30 \mathrm{~nm}$.

In our simulation, the vortex states are brought out by two different methods. The first method is to saturate an element of a large size and then to relax it in zero field. The second method is to set the direction of the magnetization of each numerical cell distributed arbitrarily. On decreasing of the total energy, the multivortex states are eventually formed. Experimentally, the 

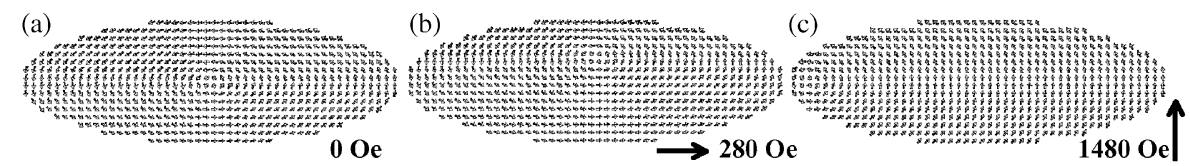

Fig. 1. Spin configurations of the one-vortex state at different applied fields. The arrows represent the intensity and direction of the field with respect to the particle's longest axis. The dimension of the element is 360,120 , and $30 \mathrm{~nm}$ in long axis, short axis, and thickness, respectively.

vortex states can be obtained by relaxation from saturation through different magnetization processes. These processes are effected by the sample shape, size, boundary irregularities, and magnetic history [2,5].

Figs. 1(a), 3(a) and 5(a) represents the typical spin configurations of the one-, two-, and three-vortex states existing in Permalloy magnetic elements. $180^{\circ}$ Neel type walls are located near the two ends of the long axis, while $90^{\circ}$ Neel type walls are along the sides of the rhombic domains, which are almost uniformly magnetized perpendicular to the longest element axis. The singularities at the center of the vortices have been observed in some experiments [6,7]. Within a radius of the exchange length around the singularities, the magnetization has a significant component perpendicular to the sample plane. The vortices form a flux closure and the total magnetization is close to zero.

In the one-vortex state the external field extends the area within which the magnetization is parallel to the field, and contracts the area magnetized in the opposite direction. Figs. 1(b) and (c) represent the spin configuration of the element under a field of 280 Oe along the long axis and of $1480 \mathrm{Oe}$ along the short axis. It is obvious that the vortex always moves in the direction perpendicular to external field. Fig. 2 represents the magnetization curves of the one-vortex state at different field angles with respect to the long axis. When the external field increases along the long axis, that is, at $\phi=0^{\circ}$ in Fig. 2, the total magnetization of the element initially increases gradually. Once the field reaches $300 \mathrm{Oe}$ the vortex is annihilated near the element boundary, and this is accompanied by a sharp increase of the total magnetization. At this moment the spin configuration becomes quasiuniform. Furthermore, if the applied field is parallel to the short axis, then the vortex moves to the boundary along the long axis. As the field reaches $1500 \mathrm{Oe}$ the total magnetization increases abruptly, and the vortex leaves the element. After that, the magnetization is continuous to increases monotonically with the field since the demagnetization field is so large that it is more difficult to saturate the element in the short axis direction. The magnetization curve for $\phi=45^{\circ}$ is shown here to illustrate the angular dependence. The magnetization process is reversible before the annihilation of the vortex regardless of the

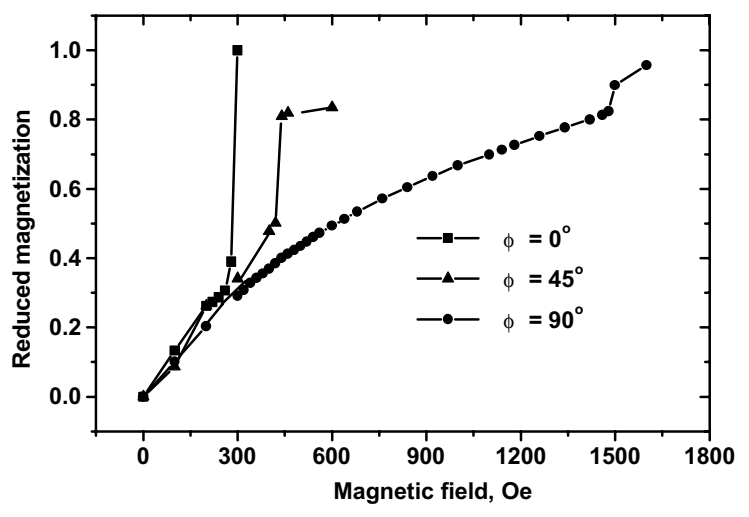

Fig. 2. Magnetization curves of the one-vortex state for different angles $\phi$ spanned by the field and the longest particle's axis.

angle $\phi$. In addition to the angular dependence, both experiments $[5,8]$ and theory [9] show that the critical field also depends on the strength of the demagnetization field related to the size and thickness of the element.

In the two-vortex state the two vortices form a rhombic domain which is almost uniformly magnetized perpendicular to the longest axis. The specific direction of the mean magnetization of the rhombic domain leads to two different kinds of magnetization processes for field parallel and anti-parallel to the mean magnetization of the rhombic domain. When the field increases along the long axis, the area where the magnetization is parallel to the field enlarges, the rhombic region is deformed, and its boundary tilts. In this case one vortex moves up and the other moves down as shown in Fig. 3(b) for the field of 100 Oe. As the field increases further to $160 \mathrm{Oe}$ an instability occurs, and the two vortices are simultaneously annihilated near the boundary of the element, giving rise to quasiuniform spin configuration. At this moment the total magnetization increases abruptly as shown in Fig. 4 for $\phi=0^{\circ}$.

When the field increases parallel to the mean magnetization of the rhombic domain, the rhombic region which is energetically favorable extends, and the two vortices move in opposite directions along the long axis as shown in Fig. 3(c) at 1000 Oe. When the field increases to $1100 \mathrm{Oe}$ one vortex leaves the element as shown in Fig. 3(d). As the field reaches 1400 Oe, the 

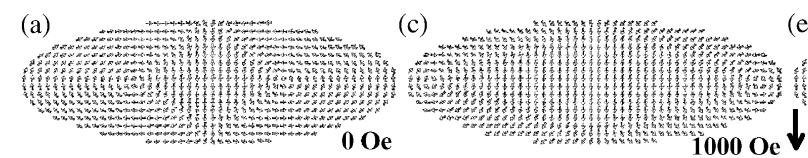

(e)
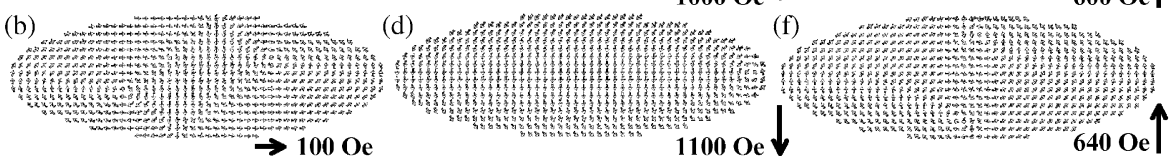

Fig. 3. Spin configurations of the two-vortex state at different applied fields. The arrows represent the intensity and direction of the field with respect to the particle's longest axis, and $\phi=90^{\circ}$ and $-90^{\circ}$ represent the field parallel and anti-parallel to the mean magnetization of the rhombic domain, respectively. The dimension of the element is 720,240 , and $30 \mathrm{~nm}$ in long axis, short axis, and thickness, respectively.

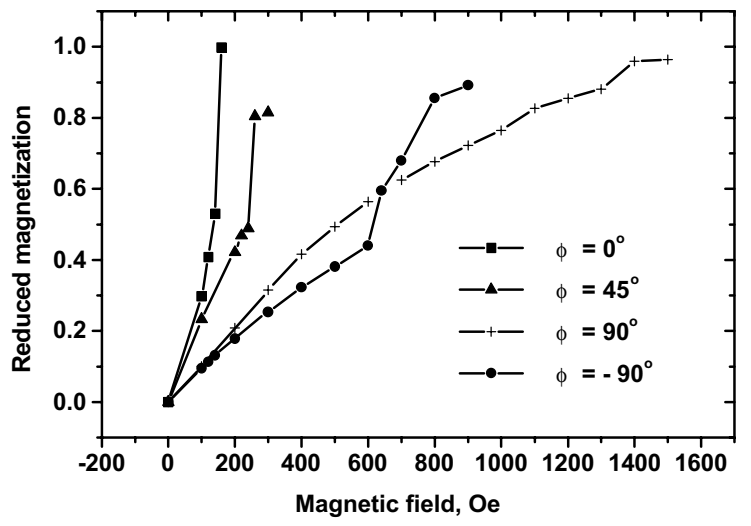

Fig. 4. Magnetization curves of the two-vortex state of different field angles.

residual vortex also leaves the element and again causes an abrupt increase of the total magnetization (see Fig. 4).

When the field increases anti-parallel to the mean magnetization of the rhombic domain the rhombic region, which is energetically unfavorable, reduces, and the two vortices move toward each other along the long axis as shown in Fig. 3(e) at 600 Oe. As the field reaches $640 \mathrm{Oe}$, the rhombic region suddenly disappears as shown in Fig. 3(f), and the magnetization curve experiences a jump as shown in Fig. 4 for $\phi=-90^{\circ}$. When the field increases further to $800 \mathrm{Oe}$, an instability occurs, and the two vortices are simultaneously annihilated near the boundary of the element. At the same time the magnetization curve experiences a second jump. In fields larger than 800 Oe the spin configuration of the element is quasiuniform, with a strong shape anisotropy effect which modifies the magnetization near the boundary.

As in the previously discussed one-vortex state the magnetization curves depend also here on the directions of the field, so that, for example, the $45^{\circ}$ magnetization curve is between the cases of $0^{\circ}$ and $90^{\circ}$. The lowest critical field occurs of the field parallel to the long axis. Also note that when the field is small the slope of the magnetization curve is larger for $\phi=90^{\circ}$ than for $\phi=-90^{\circ}$.

Lastly, we consider the three-vortex state if the external field increases in this case parallel to the long axis then the region where the magnetization is parallel to the field extends, and the middle vortex moves along the short axis in one direction, while the other two vortices move in the opposite direction. The spin configuration of $100 \mathrm{Oe}$ is shown in Fig. 5(b). If the field increases to 180 Oe the three vortices are simultaneously annihilated near the boundary of the element. At this moment the total magnetization increases significantly (see Fig. 6 for $\phi=0^{\circ}$ ), and the spin configuration becomes quasiuniform.

As the field increases along the short axis, one of the two rhombic domains with mean magnetization parallel to the applied field extends and the other one contracts. The spin configuration of $200 \mathrm{Oe}$ is shown in Fig. 5(c). At 640 Oe one of the vortices leaves the element (see Fig. 5(d)). When the field increases to $680 \mathrm{Oe}$ the residual two vortices are annihilated simultaneously. At this moment the spin configuration is strongly curved as shown in Fig. 5(e). Surprisingly, as the field reaches 740 Oe a new vortex (see Fig. 5(f)) is nucleated from the corner of the huge curving of the spin configuration at the boundary. This is accompanied by an abrupt drop of the magnetization, as shown in Fig. 6 for $\phi=90^{\circ}$. The final vortex is annihilated in fields larger than $1400 \mathrm{Oe}$, causing a final jump in the magnetization curve.

The curve in Fig. 6 marked by star symbols represents the back sweep process at fields along the short axis from 640 to $0 \mathrm{Oe}$, that is, from the point when the first vortex has annihilated from the element. It is interesting to find that the element remains in the two-vortex instead of the three-vortex state throughout the back sweep. This suggests a method to transform three-vortex states to two-vortex ones. The figure shows that the back sweep curve deviates from the original one since it follows a different vortex configuration. Also note that in the back sweep case the two vortices gradually move to the central part of the element and become a typical two-vortex state corresponding to zero magnetization. 

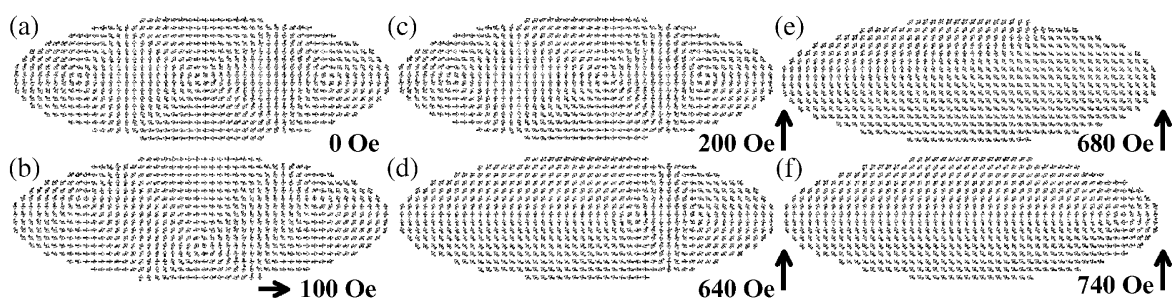

(d)

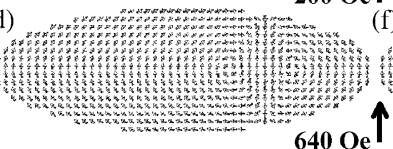

(f)

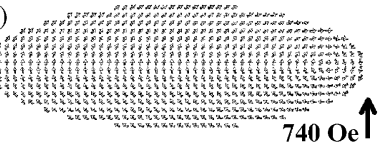

Fig. 5. Spin configurations of the three-vortex state at different applied fields. The arrows represent the intensity and direction of the field with respect to the particle's longest axis. The dimension of the element is 720,240 , and $30 \mathrm{~nm}$ in long axis, short axis, and thickness, respectively.

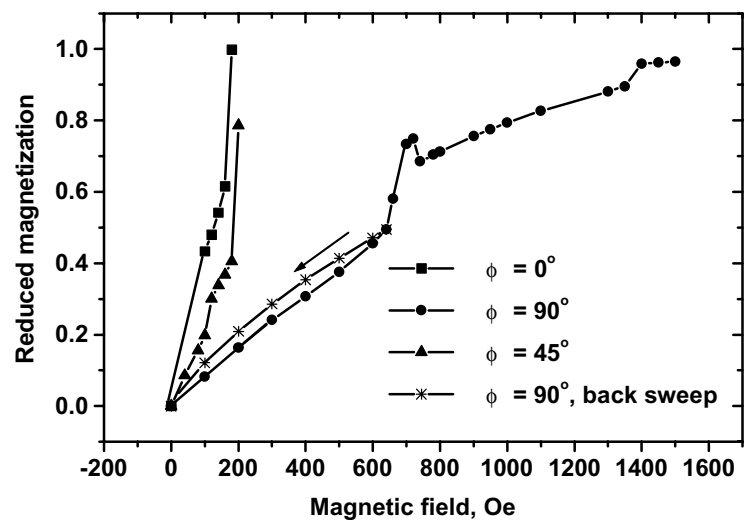

Fig. 6. Magnetization curves of the three-vortex state at different field angles. The curve with star symbols represents the back sweep process at fields along the short axis from 640 to 0 Oe.

To summarize the properties of the multi-vortex states described above, we state that odd-numbered vortices lead to even-numbered rhombic domains, and the corresponding magnetization pattern is symmetric with respect to the short axis direction, so there is only one magnetization process if the field is applied along the short axis. On the other hand, even-numbered vortices lead to odd-numbered rhombic domains, which make the corresponding magnetization pattern asymmetric with respect to the short axis. This asymmetry leads to two different kinds of magnetization processes if the field is applied along the short axis. By studying the symmetry property of the magnetization curves in opposite directions along short axis we can obtain information about the number of vortices present in the sample.

This work was supported in part by the ROC National Science Council Grant \# NSC 89-2119M002-014.

\section{References}

[1] Jian-Gang Zhu, Youfeng Zheng, Gary A. Prinz, J. Appl. Phys. 87 (2000) 6668.

[2] Luc Thomas, Stuart S.P. Parkin, Jun Yu, Ulrich Rüdiger, Andrew D. Kent, Appl. Phys. Lett. 76 (2000) 766.

[3] R.D. Gomez, T.V. Luu, A.O. Pak, K.J. Kirk, J.N. Chapman, J. Appl. Phys. 85 (1999) 6163.

[4] Jian-Gang Zhu, Youfeng Zheng, Xiangdong Lin, J. Appl. Phys. 81 (1997) 4336.

[5] A. Fernandez, C.J. Cerjan, J. Appl. Phys. 87 (2000) 1 395.

[6] T. Shinjo, T. Okuno, R. Hassdorf, K. Shigeto, T. Ono, Science 289 (2000) 930.

[7] J. Raabe, R. Pulwey, R. Sattler, T. Schweinböck, J. Zweck, D. Weiss, J. Appl. Phys. 88 (2000) 4437.

[8] A. Fernandez, M.R. Gibbons, M.A. Wall, C.J. Cerjan, J. Magn. Magn. Mater. 190 (1998) 71.

[9] Konstantin Yu. Guslienko, Konstantin L. Metlov, Phys. Rev. B 63 (2001) 100403. 